\title{
Gold Nanoparticles: 3D-STEM-HAADF Analyses and Plasmonic Studies by EELS
}

\author{
R. Arenal ${ }^{* * *}$, L. Roiban $* * *$, O. Ersen***, J. Burgin, ****, M. Treger-Delapierre**** \\ * Laboratorio Microscopias Avanzadas, INA, U. Zaragoza, 50018 Zaragoza - Spain \\ ** LEM, UMR 104 CNRS-ONERA, 92322 Châtillon - France \\ *** IPCMS, UMR 7504 CNRS-U. Strasbourg, 67087 Strasbourg - France \\ **** ICMCB, UPR 9048 CNRS, 33608 Pessac/Bordeaux - France
}

In recent years a significant interest has been developed in the study of the optical properties of noble metallic nanoparticles (NPs) due to their applications in photonics, electronics and biosensors [1]. As matter of fact, it is possible to control their optical response, for instance the surface plasmon resonances (SPRs). It is well known, that particle size, shape and composition, as well as the local dielectric environment of NPs have strong impact on these SPRs [1]. Electron tomography is a very powerful technique giving access to all these crucial structural, morphological and compositional aspects [2-5]. Thus, we have developed 3D-STEM-HAADF measurements in AuAg core-shell and Au nanoparticles obtaining such rich information.

These studies have been carried in a FEI Titan - XFEG - Cs probe corrected microscope equipped with a monochromator (working at $300 \mathrm{kV}$ ). NPs were deposited on amorphous carbon films on a $\mathrm{Cu}$ grid with repeated plasma cleaning to eliminate organic contamination prior to the STEM-tomography and EELS analyses. The tilt-tomographical series were acquired using Xplore3DFEI software. During the acquisition, defocus and XY sample shift were controlled manually, the rest of TEM parameters, as the tilt were controlled automatically. The tilt angle range was of $+/-72^{\circ}$ $\left(+/-60^{\circ}\right.$ in other cases), with an image recorded every $2^{\circ}$ in Saxton mode. After acquisition, the tilt series data were treated for imaging processing, alignment and reconstruction, using IMOD and a macro of TomoJ software programs, respectively. For the volume reconstruction we employed an algebraic reconstruction technique (15 iterations) and 3DSlicer software program has been employed for the visualization of the volume. It is worth noting that no evidence of irradiation damage in the samples was detected during the acquisition. For the low-loss studies, the energy resolution was below $250 \mathrm{meV}$ and the spectra were collected in STEM mode.

Figure 1 (a) displays the low magnification high-angle annular dark field STEM image of one of the tipical nanoparticles. Their shape is similar to a "stone-age" arrowhead and their size is around 150-170 $\mathrm{nm}$ by 50-60 nm. High-resolution HAADF-STEM image of one of the apexes of this nanoparticle is showed in Figure 1 (b). From the analysis of this image we interpret that this structure corresponds to a silver region. This result has been confirmed via EDS measurements developed in this area, as well as in other areas of the particle (see Figure 1 (c)). From the other EDS spectra it can be inferred that the nanoparticle correspond to a core-shell structure.

As mentioned above, STEM-HAADF tomography allows obtaining indirect compositional information due to the $Z$ dependence of the inelastic scattered signal. Figure 2 (a) shows the volume reconstruction of one of these NPs. From these studies we have shown that the nanoparticles have a perfect and homogeneous Au-Ag core-shell structure. Their morphology corresponds to a bipyramidal prism, composed by an Au core and covered by an Ag shell of few nm thick, as can be deduced from the sections/slices showed in Figure 2(b). Further discussions of these results will be presented in this contribution. 
Low-loss EELS measurements, developed on these nanostructures will be also discussed in this presentation. As a matter of fact, recently a significant activity in this field has been generated, due to the rich optical properties information that can be obtained from this technique $[6,7]$.

\section{References}

[1] L.M. Liz-Marzan, Langmuir 22, 32 (2006).

[2] A.J. Koster, et al. J. Phys. Chem. B 104, 9368 (2000).

[3] C. Kubel, et al. Microsc. Microanal. 11, 378 (2005).

[4] P.A. Midgley and R.E. Dunin-Borkowski, Nature Mat. 8, 271 (2009).

[5] O. Ersen, et al. Nano Lett. 7, 1898 (2007).

[6] R. Arenal, et al. Phys. Rev. Lett. 95, 127601 (2005); R. Arenal, et al. Microsc. Microanal. 11, 378 (2008).

[7] J. Nelajah, et al. Nature Phys. 3, 348 (2007).
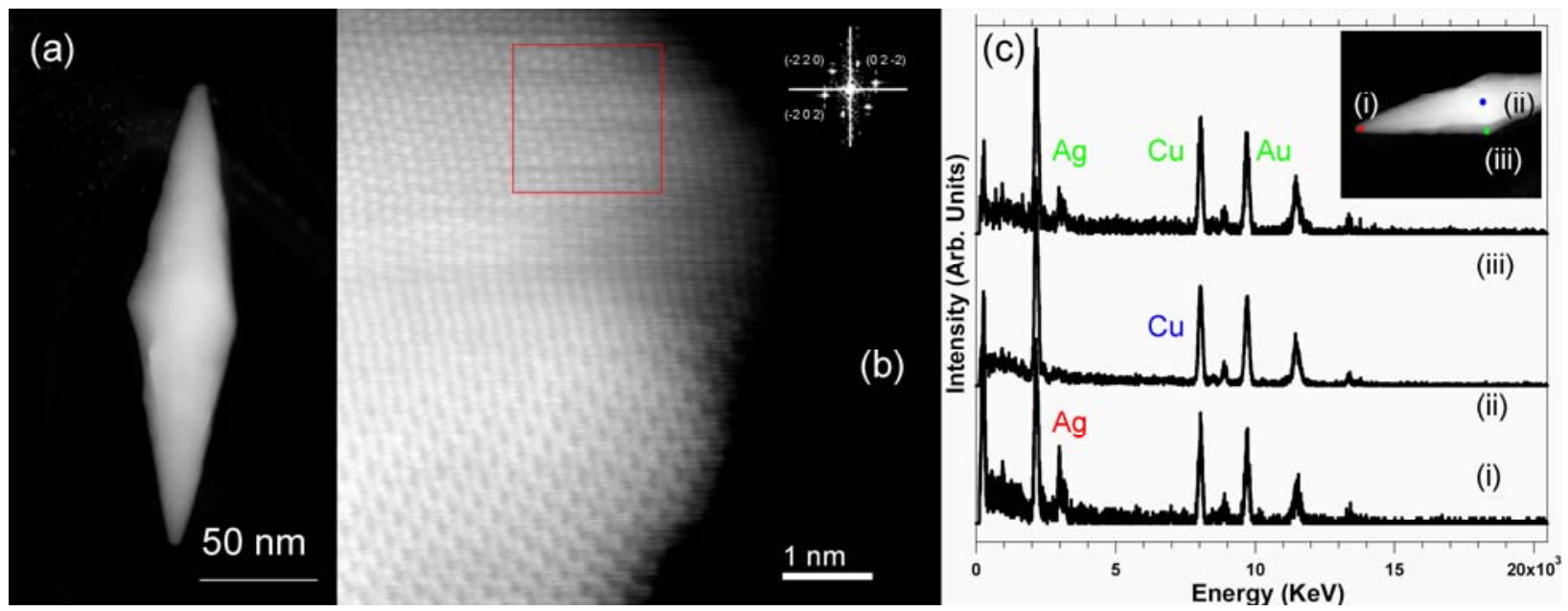

Figure 1. (a) Low magnification HAADF image of one of the Au-Ag core-shell bipyramidal nanoparticles. (b) HR-HAADF image recorded at the apex of this nanoparticle and corresponding to the $\mathrm{Ag}$ region. Inset: FFT calculated from the marked area of the HR-HAADF micrograph and that is indexed as Ag. (c) EDS spectra recorded in two different regions marked on the LM-HAADF image.
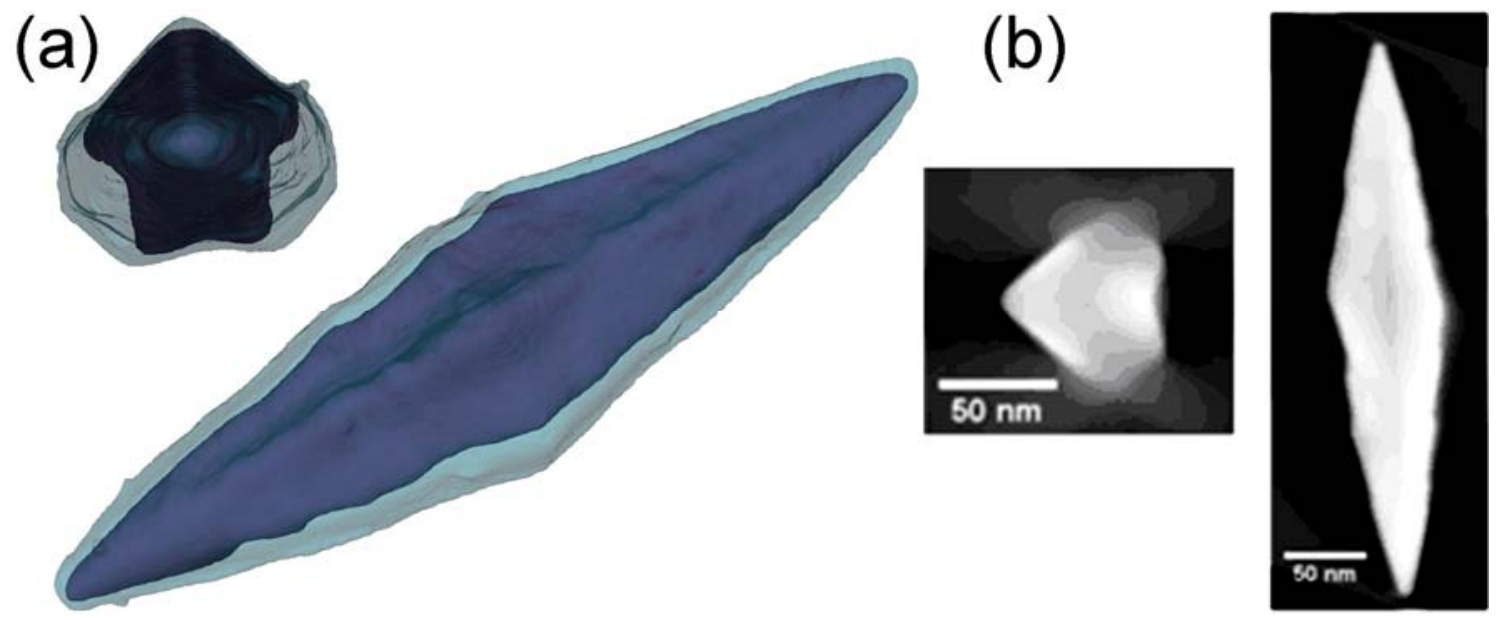

Figure 2. (a) Two different views of the tomographical reconstruction of the Au-Ag core-shell nanoparticle showing the internal gold structure and the outer silver sheath. (b) Examples of two different sections of this reconstructured volume. 